\title{
Dynamic Scaling of Ion-Sputtered Surfaces
}

\author{
Rodolfo Cuerno' ${ }^{1}$ and Albert-László Barabási ${ }^{1,2}$ \\ ${ }^{\prime}$ Center for Polymer Studies and Department of Physics, Boston University, Boston, Massachusetts 02215 \\ ${ }^{2}$ IBM T. J. Watson Research Center, P.O. Box 218, Yorktown Heights, New York 10598
}

\begin{abstract}
We derive a stochastic nonlinear equation to describe the evolution and scaling properties of surfaces eroded by ion bombardment. The coefficients appearing in the equation can be calculated explicitly in terms of the physical parameters characterizing the sputtering process. We find that transitions may take place between various scaling behaviors when experimental parameters, such as the angle of incidence of the incoming ions or their average penetration depth, are varied.
\end{abstract}

PACS numbers: $79.20 . \mathrm{Rf}, 64.60 . \mathrm{Ht}, 68.35 . \mathrm{Rh}$

Recently there has been much interest in understanding the formation and roughening of nonequilibrium interfaces [1]. A common feature of most rough interfaces observed experimentally or in discrete models is that their roughening follows simple scaling laws. The associated scaling exponents can be obtained using numerical simulations or stochastic evolution equations. The morphology and dynamics of a rough interface can be characterized by the surface width, $w(t, L)$, that scales as $w^{2}(t, L)=$ $\left\langle[h(r, t)-\bar{h}(t)]^{2}\right\rangle=L^{2 \alpha} f\left(t / L^{z}\right)$, where $\alpha$ is the roughness exponent for the interface $h(r, t)$ and the dynamic exponent $z$ describes the scaling of the relaxation times with the system size $L ; \bar{h}(t)$ is the mean height of the interface at time $t$ and \langle\rangle denotes both ensemble and space average. The scaling function $f$ has the properties $f(u \longrightarrow 0) \sim u^{2 \alpha / z}$ and $f(u \rightarrow \infty) \sim$ const.

Much of the attention has focused so far on the kinetics of interfaces generated in growth processes. However, for a class of technologically important phenomena, such as sputter etching, the surface morphology evolves as a result of erosion processes [2]. Motivated by the advances in understanding growth, recently a number of experimental studies have focused on the scaling properties of surfaces eroded by ion bombardment [3-5]. For graphite bombarded with $5 \mathrm{keV}$ Ar ions, Eklund et al. [3] reported $\alpha \approx 0.2-0.4$ and $z=1.6-1.8$, values consistent with the predictions of the Kardar-Parisi-Zhang (KPZ) equation in $2+1$ dimensions [6,7]. Krim et al. [4] observed a selfaffine surface generated by $5 \mathrm{keV}$ Ar bombardment of an Fe sample, with a larger exponent, $\alpha=0.52$. On the other hand, there exists ample evidence about the development of a periodic ripple structure in sputter-etched surfaces (see, e.g., [8]). Chason et al. [5] have recently studied the dynamics of such eroded surfaces for both $\mathrm{SiO}_{2}$ and $\mathrm{Ge}$ bombarded with $\mathrm{Xe}$ ions at $1 \mathrm{keV}$, and found that it differs from the dynamics expected for the self-affine morphologies observed in [3] and [4].

In this paper we investigate the large scale properties of ion-sputtered surfaces aiming to understand in a unified framework the various dynamic and scaling behaviors of the experimentally observed surfaces. For this we derive a stochastic nonlinear equation that describes the time evolution of the surface height. The coefficients appearing in the equation are functions of the physical parameters characterizing the sputtering process. We find that transitions may take place between various surface morphologies as the experimental parameters (e.g., angle of incidence, penetration depth) are varied. Namely, at short length scales the equation describes the development of a periodic ripple structure, while at larger length scales the surface morphology may be either logarithmically $(\alpha=0)$ or algebraically $(\alpha>0)$ rough. Usually stochastic equations describing growth models are constructed using symmetry principles and conservation laws. In contrast, here we show that for sputter eroded surfaces the growth equation can be derived directly from a simple model of the elementary processes taking place in the system.

Most of the theoretical approaches focusing on the scaling properties of sputter roughened surfaces have assumed that essentially all relevant processes take place at the surface, and that nonlinear effects would appear only due to nonlocal effects such as shadowing [9]. However, ion sputtering is in general determined by atomic processes taking place along a finite penetration depth inside the bombarded material. The incoming ions penetrate the surface and transfer their kinetic energy to the atoms of the substrate by inducing cascades of collisions among the substrate atoms, or through other processes such as electronic excitations. Whereas most of the sputtered atoms are located at the surface, the scattering events that might lead to sputtering take place within a certain layer of average depth $a$. As described by Sigmund's transport theory of sputtering [10], the average value of the ion deposition depth depends on the energy of the bombarding ions, their angle of incidence, the microscopic structure of the target material, and the features of the scattering processes taking place inside the sample.

A convenient picture of the ion bombardment process is sketched in the inset of Fig. 1. According to it the ions penetrate a distance $a$ inside the solid before they 


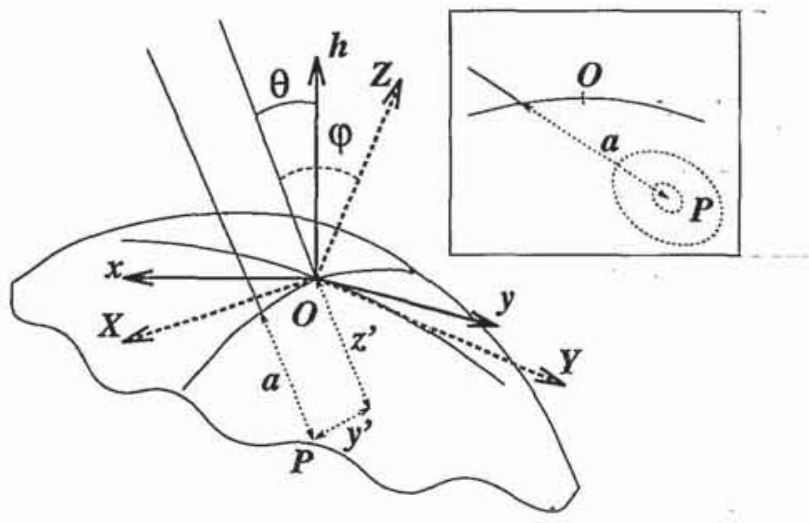

FIG. 1. Reference frames for the computation of the erosion velocity at point $O$. Inset: Following a straight trajectory (solid line) the ion penetrates an average distance $a$ inside the solid (dotted line) after which it completely spreads out its kinetic energy. The dotted curves are equal energy contours. Energy released at point $P$ contributes to erosion at $O$.

completely spread out their kinetic energy with some assumed spatial distribution. An ion releasing its energy at point $P$ in the solid contributes an amount of energy to the surface point $O$ that may induce the atoms in $O$ to break their bonds and leave the surface. Following $[10,11]$, we consider that the average energy deposited at point $O$ due to the ion arriving at $P$ follows the Gaussian distribution

$$
E\left(\boldsymbol{r}^{\prime}\right)=\frac{\epsilon}{(2 \pi)^{3 / 2} \sigma \mu^{2}} \exp \left\{-\frac{z^{\prime 2}}{2 \sigma^{2}}-\frac{x^{\prime 2}+y^{2}}{2 \mu^{2}}\right\} .
$$

In (1) $z^{\prime}$ is the distance measured along the ion trajectory, and $x^{\prime}, y^{\prime}$ are measured in the plane perpendicular to it (see Fig. 1; for simplicity in the figure $x^{\prime}$ has been set to 0$) ; \epsilon$ denotes the total energy carried by the ion and $\sigma$ and $\mu$ are the widths of the distribution in directions parallel and perpendicular to the incoming beam, respectively. However, the sample is subject to a uniform flux $J$ of bombarding ions. A large number of ions penetrate the solid at different points simultaneously, and the velocity of erosion at $O$ depends on the total power $\mathcal{E}_{O}$ contributed by all the ions deposited within the range of the distribution (1). If we ignore shadowing effects among neighboring points, as well as further redeposition of the eroded material, the normal velocity of erosion at $O$ is given by

$$
v=p \int_{\mathcal{R}} d r \Phi(r) E(r),
$$

where the integral is taken over the region $\mathcal{R}$ of all the points at which the deposited energy contributes to $\mathcal{E}_{O}$, $\Phi(r)$ is a local correction to the uniform flux $J$, and $p$ is a proportionality constant between power deposition and rate of erosion. In the following we outline the basic steps in the calculation of $v$; further details can be found in Refs. [11,12].
The calculation of (2) is most efficiently perfomed in the local coordinate system $(X, Y, Z)$ shown in Fig. 1 . The ion beam lies in the $X-Z$ plane, forming an angle $\varphi$ with the $Z$ axis, $Z$ being normal to the surface at $O$. To simplify the calculations, we assume the following: (a) the radii of curvature $\left(R_{X}, R_{Y}\right)$ of the surface at $O$ are much larger than the penetration depth $a$, so that only terms up to first order in $a / R_{X, Y}$ are kept; and (b) the curvatures attain their maximum and minimum values along either of the $X$ and $Y$ directions, and thus we can expand the value of the surface height at $O$ by taking a second order approximation in the $(X, Y)$ coordinates and consistently ignoring cross term contributions. Performing the integral (2) the velocity $v\left(\varphi, R_{X}, R_{Y}\right)$ is found to be a function of the angle $\varphi$ and the curvatures $1 / R_{X, Y}[11]$.

Finally, from the expression of $v$ we can obtain the equation of motion for the profile. Now it is convenient to use the laboratory coordinate frame $(x, y, h)$. In the absence of overhangs the surface can be described by a single valued height function $h(x, y, t)$, measured from an initial flat configuration which is taken to lie in the $(x, y)$ plane. The ion beam is parallel to the $x-h$ plane forming an angle $0<\theta<\pi / 2$ with the $z$ axis. The time evolution of $h$ is given by.

$$
\frac{\partial h(x, y, t)}{\partial t} \simeq-v\left(\varphi, R_{X}, R_{Y}\right) \sqrt{1+(\nabla h)^{2}},
$$

where $\varphi$ is the angle of the beam direction with the local normal to the surface at $h(x, y)$. Now $\varphi$ is a function of the angle of incidence $\theta$ and the values of the local slopes $\partial_{x} h$ and $\partial_{y} h$, and can be expanded in powers of the latter. We will assume that the surface varies smoothly enough so that products of derivatives of $h$ can be neglected for third or higher orders.

At this stage additional relevant physical processes must be taken into account to describe the evolution of the surface. First, the bombarding ions reach the surface at random positions and times. We account for the stochastic arrival of ions by adding to (3) a Gaussian white noise $\eta(x, y, t)$ with zero mean and variance proportional to the flux $J$. Second, at finite temperature atoms diffuse on the surface $[3,5]$. To include this surface self-diffusion we allow for a term $-K \nabla^{2}\left(\nabla^{2} h\right)$ [13], where $K$ is a temperature dependent positive coefficient. Expanding (3) and adding the noise and the surface-diffusion terms we obtain the equation of motion [14]

$$
\begin{aligned}
\frac{\partial h}{\partial t}= & -v_{0}+\gamma \frac{\partial h}{\partial x}+\nu_{x} \frac{\partial^{2} h}{\partial x^{2}}+\nu_{y} \frac{\partial^{2} h}{\partial y^{2}} \\
& +\frac{\lambda_{x}}{2}\left(\frac{\partial h}{\partial x}\right)^{2}+\frac{\lambda_{y}}{2}\left(\frac{\partial h}{\partial y}\right)^{2}-K \nabla^{2}\left(\nabla^{2} h\right)+\eta .
\end{aligned}
$$

From (3) we can compute the expressions for the coefficients appearing in (4) in terms of the physical parameters characterizing the sputtering process. To simplify the discussion we restrict ourselves to the symmetric case $\sigma=\mu$. The general case is discussed in [12]. If we 
write $F \equiv(\epsilon J p / \sqrt{2 \pi}) \exp \left(-a_{\sigma}^{2} / 2\right), s \equiv \sin \theta, c \equiv \cos \theta$, and $a_{\sigma} \equiv a / \sigma$, we find for the coefficients in (4)

$$
\begin{aligned}
& \nu_{0}=\frac{F}{\sigma} c, \quad \gamma=\frac{F}{\sigma} s\left(a_{\sigma}^{2} c^{2}-1\right), \\
& \lambda_{x}=\frac{F}{\sigma} c\left\{a_{\sigma}^{2}\left(3 s^{2}-c^{2}\right)-a_{\sigma}^{4} s^{2} c^{2}\right\}, \\
& \lambda_{y}=-\frac{F}{\sigma} c\left\{a_{\sigma}^{2} c^{2}\right\}, \\
& \nu_{x}=\frac{F}{2} a_{\sigma}\left\{2 s^{2}-c^{2}-a_{\sigma}^{2} s^{2} c^{2}\right\}, \\
& \nu_{y}=-\frac{F}{2} a_{\sigma} c^{2} .
\end{aligned}
$$

Consistent with the direction of the bombarding beam and the choice of coordinates, the terms in (4) are symmetric under $y \rightarrow-y$ but not under $x \rightarrow-x$, while for $\theta \rightarrow 0$ we get $\gamma=\xi_{x}=\xi_{y}=0, \lambda_{x}=\lambda_{y}$, and $\nu_{x}=\nu_{y}$ [15]. The equation studied in Ref. [11] corresponds to the deterministic linear version of (4), i.e., $\lambda_{x}=\lambda_{y}=\eta=0$.

If $\nu_{x}$ and $\nu_{y}$ are positive, the surface-diffusion term is expected to contribute negligibly to the relevant surface relaxation mechanism when we probe the system at increasingly large length scales. Scaling properties are then described by the anisotropic KPZ equation (AKPZ), which predicts two possible behaviors depending on the relative signs of the coefficients $\lambda_{x}$ and $\lambda_{y}[16,17]$. If $\lambda_{x} \lambda_{y}>0$, then $\alpha=0.38$ and $z=1.6$, the surface width $w(L, t)$ increases algebraically, being characterized by the exponents of the KPZ equation in $2+1$ dimensions [7]. For $\lambda_{x} \lambda_{y}<0$, the nonlinear terms $\lambda_{x}$ and $\lambda_{y}$ become irrelevant, and the width grows only logarithmically, i.e., $\alpha=0$.

In our case $\nu_{x}$ can change sign as $\theta$ and $a_{\sigma}$ are varied, while $\nu_{y}$ is always negative. The negative $\nu$ causes an instability, whose origin is the faster erosion for the bottom of a trough than for the peak of a crest, as predicted by (2) (see also Fig. 3 of Ref. [11]). An instability due to a negative surface tension is also known to take place in the Kuramoto-Sivashinsky (KS) equation [18], which is the noiseless and isotropic version of (4). It has been argued for the KS equation that in $1+1$ dimensions $\nu$ renormalizes to a positive value [19], and the large length scale behavior is described by the KPZ equation. In $2+1$ dimensions it is not completely settled whether the large distance behaviors of KS and KPZ fall in the same universality class, different approaches leading to conflicting results [20].

In contrast to the KS equation, Eq. (4) is anisotropic, and explicitly contains a noise term. The competition between surface tension and surface diffusion generates a characteristic length scale in the system $\ell_{c}=\sqrt{K /|\nu|}$, where $\nu$ is the largest in absolute value of the negative surface tension coefficients. Below we discuss a possible scenario for the scaling behavior predicted by (4) based primarily on the results available in the literature for some of its limits. The complete scaling picture should be provided by either a dynamical renormalization group analysis capable of coping with the linear instabilities present in the system or a numerical integration of (4).

The scaling behavior depends on the relative signs of $\nu_{x}, \nu_{y}, \lambda_{x}$, and $\lambda_{y}$ [21]. The variations of these coefficients as functions of $a_{\sigma}$ and $\theta$ lead to the phase diagram shown in Fig. 2.

Regions $I$ and II. - For small $\theta$ both $\nu_{x}$ and $\nu_{y}$ are negative. As discussed by Bradley and Harper [11] and experimentally studied by Chason et al. [5], a periodic structure dominates the surface morphology, with ripples oriented along the direction ( $x$ or $y$ ) which presents the largest absolute value for its surface tension coefficient. The observed wavelength of the ripples is $\lambda_{c}=2 \pi \sqrt{2} \ell_{c}$.

The large length scale behavior $\ell \gg \ell_{c}$ is expected to be different. Now both nonlinearities and the noise may become relevant. The scaling properties of the surface morphologies predicted by (4) are unknown. A possible scenario is that the $\nu$ 's renormalize to positive values, as they do for the KS equation in $1+1$ dimensions, and the large scale scaling properties of the system are described by the AKPZ equation. Then one would observe algebraic scaling in region I, where both nonlinearities have the same (negative) sign, whereas scaling would become logarithmic through an AKPZ-like mechanism in region II, where $\lambda_{x}$ and $\lambda_{y}$ have opposite signs.

Region III. - This region is characterized by a positive $\nu_{x}$ and a negative $\nu_{y}$. Now the periodic structure associated with the instability is directed along the $y$ direction and is the dominant morphology at scales $\ell \sim \ell_{c}$. Again, such an anisotropic and linearly unstable equation is unexplored in the context of growth equations. Assuming that $\nu_{y}$ renormalizes to a positive value, and that the AKPZ mechanism operates, one would expect logarithmic scaling in region III, since the nonlinear terms have opposite signs.

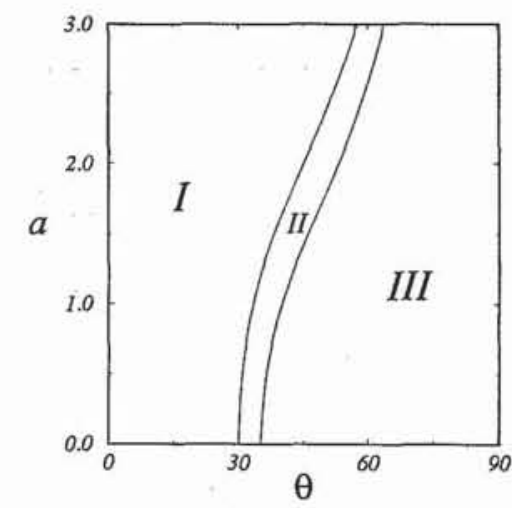

FIG. 2. Phase diagram for the isotropic case $\sigma=\mu=1$. Region I: $\nu_{x}<0, \nu_{y}<0, \lambda_{x}<0, \lambda_{y}<0$; region II: $\nu_{x}<0$, $\nu_{y}<0, \lambda_{x}>0, \lambda_{y}<0$; region III: $\nu_{x}>0, \nu_{y}<0, \lambda_{x}>0$, $\lambda_{y}<0$. Here $a$ is measured in arbitrary units and $\theta$ is measured in degrees. 
Even though several aspects of the scaling behavior predicted by (4) and (5) remain to be clarified, we believe that these equations contain the relevant ingredients for understanding roughening by ion bombardment [22]. To summarize, at short length scales the morphology consists of a periodic structure oriented along the direction determined by the largest in absolute value of the negative surface tension coefficients [5]. Modifying the values of $a_{\sigma}$ or $\theta$ changes the orientation of the ripples $[8,11]$. At large length scales we expect two different scaling regimes. One is characterized by the KPZ exponents, which might be observed in region I in Fig. 2. Indeed, the values of the exponents reported by Eklund et al. [3] are consistent within the experimental errors with the KPZ exponents in $2+1$ dimensions. The other regions (II and III) are characterized by logarithmic scaling $(\alpha=$ 0 ), which has not been observed experimentally so far. Moreover, by tuning the values of $\theta$ and/or $a_{\sigma}$ one may induce transitions among the different scaling behaviors. For example, fixing $a_{\sigma}$ and increasing the value of $\theta$ would lead from KPZ scaling (region I) to logarithmic scaling (II, III) for large enough angles.

The experimental verification of the above possibilities would constitute an important step to elucidate the interplay between the mechanisms leading to the different morphologies and dynamics for sputter-etched surfaces. It will also provide additional insight into the scaling behaviors to be expected from Eq. (4).

We would like to acknowledge discussions, comments and encouragement by L.A.N. Amaral, G. Grinstein, K. B. Lauritsen, H. Makse, L. M. Sander, and H. E. Stanley. R.C. acknowledges a postdoctoral fellowship of the Spanish Ministerio de Educación y Ciencia.

[1] Dynamics of Fractal Surfaces, edited by F. Family and T. Vicsek (World Scientific, Singapore, 1991); P. Meakin, Phys. Rep. 235, 189 (1993); T. Halpin-Healey and Y.-C. Zhang, Phys. Rep. 254, 215 (1995); A.-L. Barabási and H.E. Stanley, Fractal Concepts in Surface Growth (Cambridge University Press, Cambridge, 1995).

[2] Sputtering by Particle Bombardment, edited by R. Behrisch (Springer-Verlag, Heidelberg, 1981), Vol. I; ibid. (Springer-Verlag, Heidelberg, 1983), Vol. II.

[3] E. A. Eklund et al., Phys. Rev. Lett. 67, 1759 (1991); E. A. Eklund, E.J. Snyder, and R. S. Williams, Surf. Sci. 285, 157 (1993).

[4] J. Krim et al., Phys. Rev. Lett. 70, 57 (1993).

[5] E. Chason et al., Phys. Rev. Lett. 72, 3040 (1994); T. M. Mayer, E. Chason, and A. J. Howard, J. Appl. Phys. 76,
1633 (1994)

[6] M. Kardar, G. Parisi, and Y.-C. Zhang, Phys. Rev. Lett. 56, 889 (1986).

[7] J. M. Kim and J. M. Kosterlitz, Phys. Rev. Lett. 62, 2289 (1989); B. M. Forrest and L. Tang, J. Stat. Phys. 60, 181 (1990); J. G. Amar and F. Family, Phys. Rev. A 41, 3399 (1990); K. Moser, D.E. Wolf, and J. Kertész, Physica (Amsterdam) 178A, 215 (1991); T. Ala Nissila et al., J. Stat. Phys. 72, 207 (1993).

[8] G. Carter, B. Navinšek, and J.L. Whitton, in Ref. [2], Vol. II, p. 231.

[9] For a review see G.S. Bales et al., Science 249, 264 (1990).

[10] P. Sigmund, Phys. Rev. 184, 383 (1969); J. Mater. Sci. 8, 1545 (1973).

[11] R. M. Bradley and J.M. E. Harper, J. Vac. Sci. Technol. A 6, 2390 (1988).

[12] R. Cuerno and A.-L. Barabási (unpublished).

[13] C. Herring, J. Appl. Phys. 21, 301 (1950); W. W. Mullins, J. Appl. Phys. 28, 333 (1957); in the context of stochastic models see D. E. Wolf and J. Villain, Europhys. Lett. 13, 389 (1990); S. Das Sarma and P.I. Tamborenea, Phys. Rev. Lett. 66, 325 (1991).

[14] In the expansion of (3) two additional nonlinearities are obtained on the right-hand side of Eq. (4): $\xi_{x}(\partial h / \partial x)\left(\partial^{2} h / \partial x^{2}\right)+\xi_{y}(\partial h / \partial x)\left(\partial^{2} h / \partial y^{2}\right)$, where $\xi_{x}$ and $\xi_{y}$ are functions of $a, \sigma, \mu$, and $\theta$ [12]. One can see that $\xi_{x}$ and $\xi_{y}$ are irrelevant at large length scales using the known values of $\alpha$ and $z$ [7] for both the nonlinear and the linear fixed points in $2+1$ dimensions.

[15] The $\theta=0$ case has to be treated separately and is discussed in Ref. [12].

[16] D. E. Wolf, Phys. Rev. Lett. 67, 1783 (1991).

[17] The relevance of the AKPZ equation to sputter erosion has been suggested by R. Bruinsma, in Surface Disordering: Growth, Roughening and Phase Transitions, edited by R. Jullien et al. (Nova Science, New York, 1992).

[18] Y. Kuramoto and T. Tsuzuki, Prog. Theor. Phys. 55, 356 (1977); G. I. Sivashinsky, Acta Astronaut. 6, 569 (1979).

[19] S. Zaleski, Physica (Amsterdam) 34D, 427 (1989); K. Sneppen et al., Phys. Rev. A 46, 7352 (1992); F. Hayot, C. Jayaprakash, and Ch. Josserand, Phys. Rev. E 47, 911 (1993); V.S. L'vov et al., Nonlinearity 6, 25 (1993).

[20] I. Procaccia et al., Phys. Rev. A 46, 3220 (1992); V.S. L'vov and I. Procaccia, Phys. Rev. Lett. 69, 3543 (1992); 72, 307 (1994); C. Jayaprakash, F. Hayot, and R. Pandit, Phys. Rev. Lett. 71, 12 (1993); 72, 308 (1994).

[21] The terms $-v_{0}$ and $\partial_{x} h$ can be reabsorbed by a change of variables to a comoving frame, and do not affect the scaling properties.

[22] Our analysis describes the roughening process in the small slope approximation. However, at late stages additional nonlinear effects, such as shadowing [9], may become relevant. 